\title{
Evaluation of the furosine and homoarginine methods for determining reactive lysine in rumen-undegraded protein ${ }^{1}$
}

\author{
S. E. Boucher, ${ }^{* 2}$ C. Pedersen,$\dagger^{3}$ H. H. Stein,$\dagger^{4}$ and C. G. Schwab ${ }^{\star 5}$ \\ *Department of Biological Sciences, University of New Hampshire, Durham 03824 \\ †Department of Animal and Range Sciences, South Dakota State University, Brookings 57007
}

\begin{abstract}
Three samples of soybean meal (SBM), 3 samples of expeller SBM (SoyPlus, West Central Cooperative, Ralston, IA), 5 samples of distillers dried grains with solubles (DDGS), and 5 samples of fish meal were used to evaluate the furosine and homoarginine procedures to estimate reactive Lys in the rumen-undegraded protein fraction (RUP-Lys). One sample each of SBM, expeller SBM, and DDGS were subjected to additional heat treatment in the lab to ensure there was a wide range in reactive RUP-Lys content among the samples. Furosine is a secondary product of the initial stages of the Maillard reaction and can be used to calculate blocked Lys. Homoarginine is formed via the reaction of reactive Lys with $O$-methylisourea and can be used to calculate the concentration of reactive Lys. In previous experiments, each sample was ruminally incubated in situ for $16 \mathrm{~h}$, and standardized RUP-Lys digestibility of the samples was determined in cecectomized roosters. All rumen-undegraded residue (RUR) samples were analyzed for furosine and Lys; however, only 9 of the 16 samples contained furosine, and only the 4 unheated DDGS samples contained appreciable amounts of furosine. Blocked RUP-Lys was calculated from the furosine and Lys concentrations of the RUR. Both the intact feed and RUR samples were evaluated using the homoarginine method. All samples were incubated with an $O$-methylisourea/BaOH solution for $72 \mathrm{~h}$ and analyzed for Lys and homoarginine concentrations. Reactive Lys concentrations of the intact feeds and RUR were calculated. Results of the experiment indicate that
\end{abstract}

\footnotetext{
Received December 22, 2008.

Accepted February 23, 2009.

${ }^{1}$ This is Scientific Contribution Number 2388 from the New Hampshire Agricultural Experiment Station contributing to Regional Research Project NC-1009.

${ }^{2}$ Current address: William H. Miner Agricultural Research Institute, Chazy, NY 12921.

${ }^{3}$ Current address: Schothorst Feed Research, Lelystad, 8200 AM, the Netherlands.

${ }^{4}$ Current address: Department of Animal Sciences, University of Illinois, Champaign 61801.

${ }^{5}$ Corresponding author: charles.schwab@unh.edu
}

blocked RUP-Lys determined via the furosine method was negatively correlated with standardized RUP-Lys digestibility, and reactive RUP-Lys determined via the guanidination method was positively correlated with standardized RUP-Lys digestibility. Reactive Lys concentrations of the intact samples were also highly correlated with RUP-Lys digestibility. In conclusion, the furosine assay is useful in predicting RUP-Lys digestibility of DDGS samples, and the guanidination procedure can be used to predict RUP-Lys digestibility of SBM, expeller SBM, DDGS, and fish meal samples. Key words: reactive lysine, furosine method, homoarginine method

\section{INTRODUCTION}

Lysine is often a limiting AA for milk and milk protein production in lactating dairy cows in North America where diets high in corn products are fed (NRC, 2001). Lysine contains an $\varepsilon$-amino group on its side chain, which readily participates in the Maillard reaction in the presence of reducing sugars and heat (Hurrell and Carpenter, 1981; Mauron, 1981). The Maillard reactions result in the formation of compounds in which lysine is no longer nutritionally available but is quantified as free lysine when the standard procedure of AA analysis, including a pre-step of acid hydrolysis, is applied (Erbersdobler and Somoza, 2007). Therefore, analyzing feeds for Lys concentration using standard AA procedures is not adequate to predict the digestible Lys supply of a feed, especially if that feed has been heat processed (Moughan et al., 1996). Analysis of feeds for reactive Lys (Lys in which the $\varepsilon$-amino group is not bound) may allow for more accurate prediction of the metabolizable Lys supplied by dietary ingredients.

The reactive Lys concentration of a feed can be calculated using the furosine procedure. Furosine is an indirect measurement of $\alpha$ - $N$-formyl-( $\varepsilon$ - $N$-deoxyfrucosyl)Lys, which is the major form of blocked Lys present after the early Maillard reaction (Hurrell and Carpenter, 1981). The furosine procedure has been used primarily to evaluate lysine damage in milk products. Upon acid hydrolysis of milk products, $\alpha$ - $N$-formyl- 
( $\varepsilon$ - $N$-deoxyfrucosyl)-Lys is released as $40 \%$ Lys, $32 \%$ furosine, $10 \%$ pyridosine, and other products (Finot et al., 1981). Because these products are released in a constant ratio, the amount of furosine can be used to calculate the amount of Lys that is blocked. Reactive Lys is the amount of Lys that is not blocked. The furosine procedure has also been evaluated to estimate Lys damage in distillers dried grains with solubles (DDGS), and reactive Lys in DDGS samples determined via the furosine procedure was highly correlated with in vivo Lys digestibility (Pahm et al., 2008). In the method described by Pahm et al. (2008), the ratio of furosine to lysine generated from Amadori compounds in DDGS was assumed to be the same as in milk products.

The reactive Lys content of a feed can be determined by the homoarginine method (Moughan and Rutherfurd, 1996). For this procedure, feeds are incubated in an $O$-methylisourea/BaOH solution. $O$-Methylisourea will react with reactive Lys to form homoarginine, an amino acid not found in nature. The Lys content of the feed before and after the guanidination reaction and the amount of homoarginine formed are quantified, and reactive Lys is then calculated. These procedures have been evaluated to predict digestible Lys supply of feeds in monogastric animals (Pahm et al., 2008); however, neither the furosine procedure nor the homoarginine procedure has been evaluated to predict intestinal digestibility of Lys in the RUP fraction of feeds (RUPLys).

The objective of this experiment was to determine if the furosine and guanidination procedures can be used to accurately predict RUP-Lys digestibility of protein supplements commonly fed to lactating dairy cows. In vivo estimates of standardized RUP-Lys digestibility, determined via the precision-fed cecectomized rooster assay (Boucher et al., 2009a,b) were used to assess the adequacy of the techniques.

\section{MATERIALS AND METHODS}

Samples evaluated in this experiment were described by Boucher et al. (2009a,b). The samples evaluated were 3 samples of soybean meal (SBM), 3 samples of expeller SBM (SP; SoyPlus, West Central, Ralston, IA), 5 samples of DDGS, and 5 samples of fish meal (FM; 1 anchovy, 1 catfish, 2 menhaden, and 1 pollock) before and after a 16-h in situ ruminal incubation. One each of the SBM, SP, and DDGS samples were heated to reduce Lys digestibility. In vivo Lys digestibility in the intact samples and Lys digestibility in the rumen-undegraded residues (RUR), which represents RUP-Lys digestibility, was determined using the precision-fed cecectomized rooster assay (Boucher et al., 2009a,b). Details of the heating procedures, the ruminal incubation procedure, and the chemical composition of the intact feed and RUR samples were also described (Boucher et al., 2009a,b). All intact feed and RUR samples were ground to pass a 1-mm screen using a Wiley mill (Thomas Scientific, Swedesboro, NJ) before analysis.

\section{Furosine Analysis}

The furosine procedure described by Pahm et al. (2008) was used in this experiment. Approximately 0.2 $\mathrm{g}$ of each RUR sample was acid-hydrolyzed in $30 \mathrm{~mL}$ of $6 \mathrm{M} \mathrm{HCl}$ followed by $24 \mathrm{~h}$ of refluxing (West type reflux condensers, 24/40 S.T. joint, 300-mm jacket; Chemglass, Vineland, NJ; AOAC, 2000; method 994.12). After $24 \mathrm{~h}$, flasks were removed from the reflux condensers and cooled at room temperature $\left(22^{\circ} \mathrm{C}\right)$. The solution was transferred quantitatively to a $100-\mathrm{mL}$ volumetric flask that contained $400 \mu \mathrm{L}$ of a $31.25 \mu \mathrm{M}$ / $\mathrm{mL}$ norleucine (N8513, Sigma, St. Louis, MO) internal standard solution. The solution was made to volume, mixed thoroughly, and filtered through no. 3 filter paper (Whatman International Ltd., Maidstone, UK) into a sample jar. Five milliliters of the filtered sample was transferred to a $125-\mathrm{mL}$ round-bottomed flask and evaporated in a water bath at $50^{\circ} \mathrm{C}$ (Rotavapor R-205, Büchi, Flawil, Switzerland). At the end of the second evaporation, flasks were placed on ice to cool. Once cool, $2.5 \mathrm{~mL}$ of sodium diluent (Na220, Pickering Laboratories, Mountain View, CA) was added to the flasks and mixed well. Eight hundred fifty microliters of the solution was then filtered through a $0.45-\mu \mathrm{m}$ membrane filter (Gelman Sciences, Ann Arbor, MI) into a microcentrifuge tube and frozen at $-80^{\circ} \mathrm{C}$. Samples were analyzed for Lys and furosine concentration by ion-exchange HPLC and quantified by postcolumn derivatization using ninhydrin (South Dakota State University, Brookings). $\varepsilon-N-2$-Furomethyl-lysine (Neosystems Laboratory, Strasbourg, France) was used to quantify the furosine peak in the chromatogram at 570 nm. Blocked RUP-Lys as a percentage of total RUPLys was calculated as follows: blocked Lys $(\%)=\{[3.1$ $\times$ furosine, $\%] /[$ Lys, $\%+(1.86 \times$ furosine, $\%)]\} \times 100$ (Finot et al., 1981). The coefficient in the numerator (3.1) is the inverse of $32 \%$, and the coefficient in the denominator (1.86) is calculated based on the assumption that $60 \%$ of the lysine that is part of an Amadori compound will not be regenerated after acid hydrolysis ( $60 \%$ of $3.1 \times$ furosine).

\section{Homoarginine Analysis}

Preparation of the O-Methylisourea Solution. The guanidination reaction and subsequent analysis 
of the samples for homoarginine content were conducted according to the procedures of Moughan and Rutherfurd (1996) and Pahm et al. (2008). An Omethylisourea solution was prepared by adding $20.6 \mathrm{~g}$ of $\mathrm{BaOH}$ (217573, Sigma) to $69 \mathrm{~mL}$ of degassed water (distilled water boiled for $30 \mathrm{~min}$ ) that was cooled to $25^{\circ} \mathrm{C}$. The solution was then heated to $95^{\circ} \mathrm{C}$, and 10.4 $\mathrm{g}$ of $\mathrm{O}$-methylisourea (M53701, Sigma) was added to the solution. The solution was stirred using an automatic stirrer and allowed to cool to $25^{\circ} \mathrm{C}$. The solution was then transferred equally to 4 centrifuge tubes (50$\mathrm{mL}$ ) and centrifuged (Damon, Needham Heights, MA) at $5,000 \times g$ for $15 \mathrm{~min}$. The supernatant was then transferred to a $100-\mathrm{mL}$ beaker and $2 \mathrm{~mL}$ of degassed water was added to each centrifuge tube. The water was mixed thoroughly with the precipitate using a glass rod. The solution was centrifuged again at $5,000 \times g$ for $15 \mathrm{~min}$, and the supernatant was transferred to the beaker with the previous supernatant. The precipitate was discarded, and the $\mathrm{pH}$ of the supernatant solution was measured. If the $\mathrm{pH}$ was $<12$, the solution was discarded (Moughan and Rutherfurd, 1996; Pahm et al., 2008). If the $\mathrm{pH}$ was $>12$, the solution was adjusted to $\mathrm{pH} 11.4$ with $1 \mathrm{M} \mathrm{HCl}$ and brought to volume in a 100-mL volumetric flask.

Guanidination Reaction. For the guanidination reaction, approximately $0.2 \mathrm{~g}$ of both the intact feed and RUR samples were weighed in duplicate into $125-\mathrm{mL}$ round-bottomed flasks. Six milliliters of the $O$-methylisourea solution described above was added to each flask. A small stir rod was then added and the flasks were covered with a laboratory sealing film (DuraSeal, Diversified Biotech, Boston, MA). Samples were stirred gently via an automatic stirrer for $12 \mathrm{~h}$ at room temperature $\left(22^{\circ} \mathrm{C}\right)$. After $12 \mathrm{~h}$, the stir plate was turned off, and the samples were left to stand for 60 additional hours at room temperature. The $O$-methylisourea solution was then evaporated from the flasks. Once dry, the samples were subjected to acid hydrolysis using the procedure described above for furosine analysis, and the Lys and homoarginine content of the guanidinated samples was determined by ion-exchange HPLC and quantitated by postcolumn derivatization using ninhydrin (South Dakota State University, Brookings). Homoarginine hydrochloride (Sigma) was used as a standard to quantify the area of the homoarginine peak in the chromatograph. On the chromatograph, reactive Lys appears as homoarginine. Reactive Lys as a percentage of total Lys was calculated as follows (Pahm et al., 2008): reactive Lys $(\%)=[\mathrm{mmol}$ of homoarginine/(mmol of homoarginine + mmol of Lys $)]$ $\times$ 100. Reactive RUP-Lys was calculated similarly but was expressed as a percentage of total RUP-Lys.

\section{Statistical Analysis}

The REG procedure of SAS (SAS Institute, 2001) was used to examine the relationship between blocked and reactive Lys content and standardized Lys and RUP-Lys digestibility of the samples measured in cecectomized roosters. To determine if a mean or linear bias was present in the regression model, the residuals (observed - predicted) were evaluated against predicted values (St-Pierre, 2003) using the REG procedure of SAS (SAS Institute, 2001). Before each regression analysis, the predicted values were centered at the mean predicted values for each model because this yields a more exact test for the mean bias (St-Pierre, 2003). To center the predicted values, the mean predicted value was subtracted from the individual predicted values.

\section{RESULTS AND DISCUSSION}

\section{Furosine Analysis}

Only the RUR samples were analyzed via the furosine method. Upon furosine analysis of the RUR samples, it was determined that only 9 of the 16 samples contained any furosine, and only the 4 unheated DDGS samples contained appreciable amounts of furosine $(>0.20 \mathrm{mg} / \mathrm{g}$ of DM; Table 1). Therefore, intact samples were not analyzed for furosine content because it appeared that this assay would only be useful to determine blocked Lys content of DDGS samples. The objective of this experiment was to identify an in vitro assay that could be used to quantify reactive Lys content in a variety of feedstuffs.

Rumen undegraded residue generated from the feed samples heated in the laboratory oven did not contain any furosine (Table 1), and RUP-Lys digestibility was severely reduced for these samples (Boucher et al., $2009 a, b)$. Furosine is a product that is released upon acid hydrolysis of feeds that contain $\alpha-N$-formyl- $(\varepsilon-$ $N$-deoxyfrucosyl)-Lys, the major Amadori compound formed during the early Maillard reaction between reactive Lys and glucose, lactose, or maltose (GuerraHernandez and Corzo, 1996). During the advanced and late stages of the Maillard reaction, the Amadori compounds undergo additional reactions to form more advanced Maillard products (Mauron, 1981). Therefore, for the samples that were heated in the laboratory oven, it is likely that the prolonged heating resulted in complete conversion of Amadori compounds to more advanced Maillard reaction products. This hypothesis is supported by the fact that the heated samples contained much less reactive Lys compared with unheated samples when analyzed via the homoarginine procedure (Tables 2 and 3 ). 
Table 1. Furosine, RUP-Lys, ${ }^{1}$ and blocked RUP-Lys concentrations of samples of SoyPlus, ${ }^{2}$ soybean meal, distillers dried grains with solubles (DDGS), and fish meal (FM) samples after a 16-h ruminal incubation (DM basis)

\begin{tabular}{lccc}
\hline Rumen residue sample ${ }^{3}$ & Furosine in RUP, mg/g & RUP-Lys, mg/g & Blocked RUP-Lys, ${ }^{4}$ \\
\hline Heated SoyPlus & 0.00 & 17.2 & - \\
SoyPlus 1 & 0.00 & 30.1 & 0.10 \\
SoyPlus 2 & 0.01 & 30.3 & -1.2 \\
Heated soybean meal & 0.00 & 29.7 & 1.0 \\
Soybean meal 1 & 0.07 & 34.1 & - \\
Soybean meal 2 & 0.12 & 5.0 & 26.0 \\
Heated DDGS & 0.00 & 5.2 & 7.6 \\
DDGS 2 & 0.52 & 8.2 & 15.3 \\
DDGS 3 & 0.21 & 6.2 & 21.0 \\
DDGS 4 & 0.34 & 6.3 & 0.4 \\
DDGS 5 & 0.49 & 37.7 & - \\
Anchovy FM & 0.05 & 26.6 & 0.2 \\
Catfish FM & 0.00 & 36.9 & - \\
Menhaden FM 1 & 0.02 & 37.5 & - \\
Menhaden FM 2 & 0.00 & 37.3 & \\
Pollock FM & 0.00 & & \\
\hline
\end{tabular}

${ }^{1}$ RUP-Lys = Lys in rumen-undegraded protein.

${ }^{2}$ West Central (Ralston, IA).

${ }^{3}$ Numbers following samples indicate that these samples are from different sources or batches. Heated samples were independent samples and do not correspond to another sample.

${ }^{4}$ Blocked RUP-Lys as a percentage of total RUP-Lys.

As mentioned previously, only the 4 unheated DDGS samples contained appreciable amounts of furosine (Table 1). The other samples contained either no furosine (heated SP, SP1, heated SBM, catfish FM, menhaden FM 2, and pollock FM) or minimal amounts of furosine $(<0.20 \mathrm{mg} / \mathrm{g}$ of DM; SP2, SBM1, SBM2, anchovy FM, and menhaden FM 1). Of the samples that did contain furosine, the furosine content ranged from 0.01 to $0.52 \mathrm{mg} / \mathrm{g}$, which corresponded to blocked RUP-Lys concentrations of 0.1 and $26.0 \%$ of total RUP-Lys, respectively. Despite the limited number of samples that contained furosine $(\mathrm{n}=9)$, blocked RUP-Lys was inversely correlated with standardized RUP-Lys digestibility measured in cecectomized roosters $\left(\mathrm{R}^{2}=0.94\right.$; Figure 1). However, it is suggested that among the samples evaluated, this procedure will only be useful in estimating heat damage to RUP-Lys resulting from the early Maillard reaction in DDGS samples.

Table 2. Homoarginine, Lys, and reactive Lys concentrations of samples of SoyPlus, ${ }^{1}$ soybean meal, distillers dried grains with solubles (DDGS), and fish meal (FM) samples (DM basis)

\begin{tabular}{lccc}
\hline Intact sample $^{2}$ & Homoarginine, $\mathrm{mg} / \mathrm{g}$ & Lysine, ${ }^{3} \mathrm{mg} / \mathrm{g}$ & Reactive Lys, ${ }^{4} \%$ \\
\hline Heated SoyPlus & 5.57 & 6.54 & 37.7 \\
SoyPlus 1 & 28.85 & 1.64 & 92.5 \\
SoyPlus 2 & 22.45 & 2.96 & 84.3 \\
Heated soybean meal & 8.57 & 6.55 & 48.1 \\
Soybean meal 1 & 34.55 & 1.75 & 93.3 \\
Soybean meal 2 & 30.95 & 2.43 & 90.0 \\
Heated DDGS & 0.80 & 1.43 & 81.3 \\
DDGS 2 & 4.93 & 0.81 & 86.3 \\
DDGS 3 & 5.68 & 0.64 & 74.2 \\
DDGS 4 & 4.34 & 1.07 & 69.1 \\
DDGS 5 & 3.11 & 0.99 & 87.4 \\
Anchovy FM & 50.05 & 5.12 & 74.2 \\
Catfish FM & 33.95 & 8.36 & 87.9 \\
Menhaden FM 1 & 49.35 & 4.83 & 87.3 \\
Menhaden FM 2 & 48.90 & 5.05 & 88.7 \\
Pollock FM & 47.20 & 4.25 & \\
\hline
\end{tabular}

${ }^{1}$ West Central (Ralston, IA).

${ }^{2}$ Numbers following samples indicate that these samples are from different sources or batches. Heated samples were independent samples and do not correspond to another sample.

${ }^{3}$ Lysine concentration in sample after guanidination reaction.

${ }^{4}$ Reactive Lys as a percentage of total Lys. 


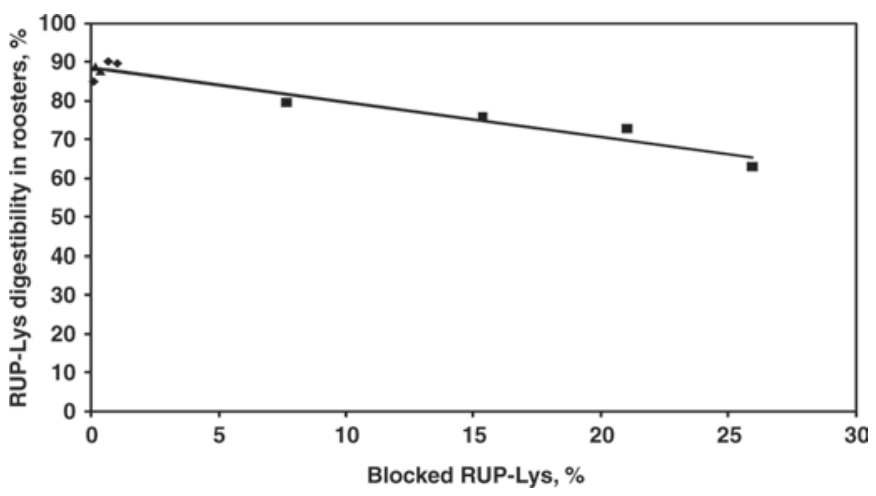

Figure 1. Regression plot of blocked RUP-Lys calculated from furosine analysis, and standardized RUP-Lys digestibility measured in cecectomized roosters $[\mathrm{Y}=88.43( \pm 1.06)-0.89( \pm 0.08) \mathrm{X}$; root mean square error (RMSE) $\left.=2.43 ; \mathrm{R}^{2}=0.94 ; P<0.001, \mathrm{n}=9\right]$ of soy product $(\bullet \mathrm{n}=3)$, distillers dried grains with solubles $(\mathbf{\square} ; \mathrm{n}=4)$, and fish meal $(\mathbf{\Lambda} ; \mathrm{n}=2)$ samples.

Furosine analysis is commonly used to assess heat damage in milk-based products (Erbersdobler and Somoza, 2007). The use of furosine to assess heat damage of animal feeds is limited, and to our knowledge, this is the first experiment to assess the furosine content in the RUP fraction of feeds. Among the unheated DDGS samples, the average blocked RUP-Lys content was (mean $\pm \mathrm{SD}) 18 \pm 7 \%$ of total RUP-Lys. Pahm et al. (2008) used the furosine procedure to estimate blocked Lys content of 33 intact DDGS samples, and the average blocked Lys content of the samples was $16 \%$ with a coefficient of variation of $7 \%$. Pahm et al. (2008) also reported that blocked Lys was correlated with standardized ileal Lys digestibility in swine $\left(\mathrm{R}^{2}=\right.$ $0.66)$. The average blocked RUP-Lys concentration of unheated DDGS samples presented in this study was similar to average blocked Lys concentrations reported by Pahm et al. (2008). However, based on results reported in this experiment and by Pahm et al. (2008), there is considerable variation in the blocked Lys content among DDGS samples. This was expected because Lys content and digestibility among DDGS samples is also highly variable (Stein et al., 2006; Kononoff et al., 2007; Boucher et al., 2009a,b). Early Maillard products will result in lower Lys concentration and lower Lys digestibility.

\section{Homoarginine Procedure}

The homoarginine concentration, lysine content after the guanidination reaction, and reactive Lys content of the intact and RUR samples are presented in Tables 2 and 3, respectively. As expected, heating the SBM, SP, and DDGS samples decreased reactive Lys and reactive RUP-Lys concentrations compared with the unheated samples. The reactive Lys concentration of the samples ranged from 28.3 (heated DDGS) to $93.3 \%$ (SBM 2) of total Lys, and the reactive RUP-Lys ranged from 31.5 (heated DDGS) to $89.4 \%$ (pollock FM) of total RUPLys. For most samples, the reactive Lys content of the intact feed was higher than the reactive Lys content of the RUR. This is likely because unreactive Lys is less likely to be degraded by rumen microbes than reactive Lys, and the concentration of unreactive Lys in the RUR is, therefore, elevated.

Table 3. Homoarginine, Lys, and reactive Lys concentrations of samples of SoyPlus, ${ }^{1}$ soybean meal, distillers dried grains with solubles (DDGS), and fish meal (FM) samples after a 16-h ruminal incubation (DM basis)

\begin{tabular}{lccc}
\hline Rumen residue sample $^{2}$ & Homoarginine, $\mathrm{mg} / \mathrm{g}$ & RUP-Lys, ${ }^{3} \mathrm{mg} / \mathrm{g}$ & Reactive RUP-Lys, $^{4} \%$ \\
\hline Heated SoyPlus & 8.36 & 10.32 & 36.5 \\
SoyPlus 1 & 35.64 & 5.79 & 81.5 \\
SoyPlus 2 & 32.51 & 6.73 & 77.4 \\
Heated soybean meal & 11.76 & 10.92 & 85.3 \\
Soybean meal 1 & 35.52 & 4.36 & 85.1 \\
Soybean meal 2 & 41.31 & 5.14 & 31.5 \\
Heated DDGS & 2.69 & 4.14 & 70.3 \\
DDGS 2 & 5.75 & 1.73 & 73.7 \\
DDGS 3 & 9.28 & 1.99 & 71.3 \\
DDGS 4 & 7.90 & 2.00 & 84.9 \\
DDGS 5 & 6.81 & 1.95 & 71.3 \\
Anchovy FM & 58.29 & 7.35 & 79.5 \\
Catfish FM & 25.06 & 7.15 & 89.1 \\
Menhaden FM 1 & 50.55 & 9.24 & 9.12 \\
Menhaden FM 2 & 48.62 & 4.94 & \\
Pollock FM & 58.49 & & \\
\hline
\end{tabular}

${ }^{1}$ West Central (Ralston, IA).

${ }^{2}$ Numbers following samples indicate that these samples are from different sources or batches. Heated samples were independent samples and do not correspond to another sample.

${ }^{3}$ Lysine concentration in rumen-undegraded residue sample after guanidination reaction.

${ }^{4}$ Reactive RUP-Lys as a percentage of total RUP-Lys. 

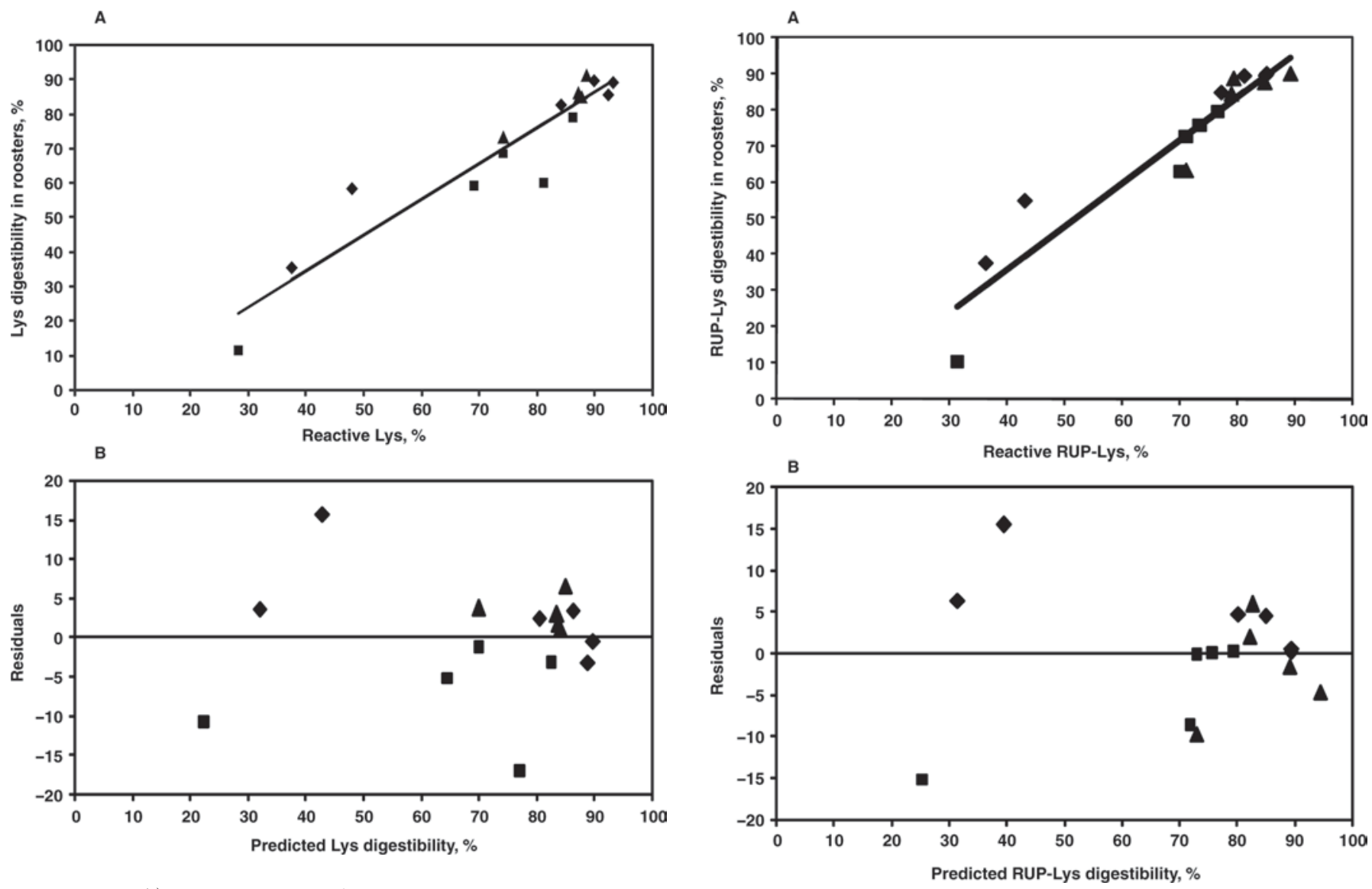

Figure 2. A) Regression plot of reactive Lys and Lys digestibility measured in cecectomized roosters $[\mathrm{Y}=-7.07( \pm 7.56)+1.04( \pm 0.10)$ $\mathrm{X}$; root mean square error $(\mathrm{RMSE})=7.56 ; \mathrm{R}^{2}=0.89 ; P<0.001, \mathrm{n}=$ $16]$, and $\mathrm{B}$ ) plot of the residuals versus predicted values $[\mathrm{Y}=-0.0019$ $( \pm 1.89)+3.05 \mathrm{E}^{-5}( \pm 0.09)(\mathrm{X}-71.4) ; \mathrm{RMSE}=7.56 ; \mathrm{R}^{2}=0.00 ; P=$ $0.99, \mathrm{n}=16]$ of soy product $(\bullet ; \mathrm{n}=6)$, distillers dried grains with solubles $(\mathbf{\square} ; \mathrm{n}=5)$, and fish meal $(\mathbf{\Lambda} ; \mathrm{n}=5)$ samples. The independent variable predicted RUP-Lys digestibility was centered around the mean predicted value before the residuals were regressed on the predicted values.

Reactive Lys concentration of the intact feeds was highly correlated with standardized Lys digestibility measured in cecectomized roosters $\left(\mathrm{R}^{2}=0.89\right.$; Figure 2 ), and the reactive RUP-Lys concentration in the RUR samples was highly correlated with in vivo RUPLys digestibility $\left(\mathrm{R}^{2}=0.90\right.$; Figure 3$)$. To determine if a mean or linear bias was present in the regression model, the residuals (observed - predicted) were plotted against centered predicted Lys and RUP-Lys digestibility values (Figures 2 and 3, respectively). The mean predicted values used to center the data were 71.4 and $71.5 \%$ for Lys and RUP-Lys, respectively. The mean bias and slope bias were nonsignificant for both Lys and RUP-Lys digestibility. Therefore, we conclude that the homoarginine procedure is an accurate procedure to estimate digestibility of Lys and RUP-Lys in SBM, SP, DDGS, and FM. Based on visual assessment of the

Figure 3. A) Regression plot of reactive RUP-Lys and RUP-Lys digestibility measured in cecectomized roosters $[\mathrm{Y}=-12.15( \pm 7.93)+$ $1.19( \pm 0.11) \mathrm{X}$; root mean square error $(\mathrm{RMSE})=7.50 ; \mathrm{R}^{2}=0.90 ; P$ $<0.001, \mathrm{n}=16)$, and $\mathrm{B}$ ) plot of the residuals versus predicted values $\left[\mathrm{Y}=-0.0002( \pm 1.88)-0.0001( \pm 0.09)(\mathrm{X}-71.2) ; \mathrm{RMSE}=7.50 ; \mathrm{R}^{2}\right.$ $=0.00 ; P=0.99, \mathrm{n}=16]$ of soy product $(\bullet ; \mathrm{n}=6)$, distillers dried grains with solubles $(\mathbf{\square} ; \mathrm{n}=5)$, and fish meal $(\boldsymbol{\Lambda} ; \mathrm{n}=5)$ samples. The independent variable predicted RUP-Lys digestibility was centered around the mean predicted value before the residuals were regressed on the predicted values.

regression plots, reactive Lys or RUP-Lys concentration determined via the homoarginine procedure will not precisely predict Lys or RUP-Lys digestibility for every sample, but, on average, the homoarginine procedure will yield an accurate estimate of Lys digestibility.

To our knowledge, this is the first experiment to estimate the reactive Lys content of RUR. Reactive Lys concentrations of intact SBM have been reported. Rutherfurd et al. (1997) reported the reactive Lys content of SBM using 2 different methods: the homoarginine and the 1-fluoro-1,4-dinitrobenzene (FDNB) procedures. The reactive Lys content of SBM was 100 and $84 \%$ determined with the homoarginine and FDNB methods, respectively, and ileal digestibility of the sample determined in swine was $95 \%$. The authors indicated that the homoarginine method cannot theoretically overestimate 

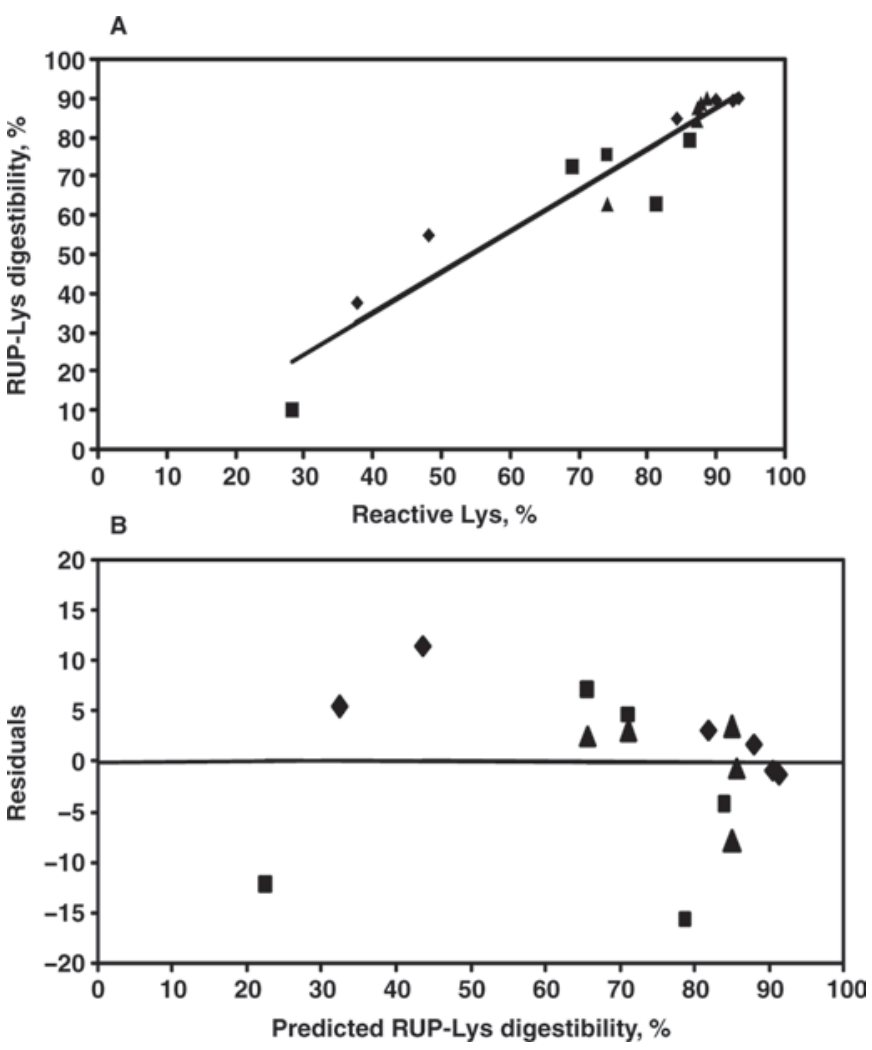

Figure 4. A) Regression plot of reactive Lys content of the intact feeds and RUP-Lys digestibility measured in cecectomized roosters $[\mathrm{Y}$ $=-7.47( \pm 7.32)+1.06( \pm 0.09) \mathrm{X}$; root mean square error $(\mathrm{RMSE})=$ $\left.7.32 ; \mathrm{R}^{2}=0.90 ; P<0.001, \mathrm{n}=16\right]$, and $\mathrm{B}$ ) plot of the residuals versus predicted values $\left[\mathrm{Y}=-7.69 \mathrm{E}^{-7}( \pm 1.83)+0.3 \mathrm{E}^{-3}( \pm 0.09)(\mathrm{X}-72.6)\right.$; $\left.\mathrm{RMSE}=7.32 ; \mathrm{R}^{2}=0.00 ; P=0.99, \mathrm{n}=16\right]$ of soy product $(\bullet ; \mathrm{n}=6)$, distillers dried grains with solubles $(\mathbf{\square} ; \mathrm{n}=5)$, and fish meal $(\mathbf{\Lambda} ; \mathrm{n}=$ 5) samples. The independent variable predicted RUP-Lys digestibility was centered around the mean predicted value before the residuals were regressed on the predicted values.

reactive Lys because the formation of homoarginine is specific to $\varepsilon$-amino groups of Lys. Based on this and other limitations of the FDNB method, Rutherfurd et al. (1997) concluded that the homoarginine procedure is preferred for estimating the reactive Lys content of feeds. The reactive Lys content of SBM determined by the homoarginine procedure in the present experiment was 90 to $93 \%$, which is lower than that reported by Rutherfurd et al. (1997). However, in vivo standardized digestibility of both unheated SBM samples was also lower (89\%; Boucher et al., 2009a). Faldet et al. (1992) determined the reactive Lys concentration of raw and heated soybeans using the FDNB method. The authors heated the soybeans at various temperatures and for various lengths of time including $150^{\circ} \mathrm{C}$ for $90 \mathrm{~min}$, which was the heat treatment applied to the heated $\mathrm{SP}$ and SBM samples in the present study. For this treatment, the authors reported that the reactive Lys content was $64 \%$ of total Lys. In the present experi- ment, the reactive Lys contents of the intact heated SP and SBM samples were 38 and $48 \%$, respectively. The discrepancy in reported values is likely because Faldet et al. (1992) evaluated whole heated soybeans, not SBM, and used a different method to measure reactive Lys.

Reactive Lys concentrations of intact DDGS samples have also been reported. Using the homoarginine method, Pahm et al. (2008) reported that the average reactive Lys concentration of 33 DDGS samples was $75 \%$ of total Lys with a coefficient of variation of $17 \%$. In the present experiment, the average reactive Lys concentration of the unheated intact DDGS samples was (mean \pm SD) $78 \pm 7 \%$ of total Lys. Pahm et al. (2008) reported that the reactive Lys content of DDGS determined via the homoarginine method was highly correlated with the standardized ileal digestible Lys content $\left(\mathrm{R}^{2}=0.70\right)$ in pigs, which agrees with the results reported here. To our knowledge, the reactive Lys content of FM has not been reported.

The relationship between the reactive Lys content of the intact samples and RUP-Lys digestibility was examined to determine if the reactive Lys concentration of the feed could be used to predict RUP-Lys digestibility. The reactive Lys content of the intact feeds was highly correlated with RUP-Lys digestibility $\left(\mathrm{R}^{2}=0.90\right.$; Figure 4). Based on these results, it is recommended that for future analyses using this method, the reactive Lys concentration of the intact feed be determined and used to predict RUP-Lys digestibility.

\section{CONCLUSIONS}

The furosine method appears to be an adequate method to predict RUP-Lys digestibility of DDGS samples. The furosine method does not appear to be useful in routinely estimating RUP-Lys digestibility in soy products or FM samples because furosine was not present in many of the samples evaluated. In addition, the application of the furosine procedure to more severely heat-damaged feeds is limited because this procedure can be used only to estimate damage from the early Maillard reaction. However, the homoarginine procedure can be used to predict Lys and RUP-Lys digestibility in a variety of feedstuffs. Further evaluation of the homoarginine procedure with similar and different feedstuffs is warranted and should focus on measuring the reactive Lys content of intact feed samples, which can be used to predict RUP-Lys digestibility. Routine analysis of feeds to determine reactive Lys will allow nutritionists and producers to more accurately meet the Lys requirements of their animals, which are particularly important for lactating dairy cows because RUP-Lys digestibility estimates reported in the litera- 
ture are limited, and Lys is a limiting AA for milk and milk protein production.

\section{ACKNOWLEDGMENTS}

The authors thank West Central (Ralston, IA) and Adisseo (Antony, France) for financial support of this project. We also thank Ameer A. Pahm, Deon Simon, and Lawrence Novotny (South Dakota State University, Brookings) for their assistance on this project.

\section{REFERENCES}

AOAC. 2000. Official Methods of Analysis. Vol. 1 and 2. 17th ed. AOAC Int., Gaithersburg, MD.

Boucher, S. E., S. Calsamiglia, C. M. Parsons, H. H. Stein, M. D. Stern, P. S. Erickson, P. L. Utterback, and C. G. Schwab. 2009a. Intestinal digestibility of amino acids in rumen undegraded protein: I. Soybean meal and SoyPlus. J. Dairy Sci. Accepted. doi:10.3168/jds.2008-1884

Boucher, S. E., S. Calsamiglia, C. M. Parsons, H. H. Stein, M. D. Stern, P. S. Erickson, P. L. Utterback, and C. G. Schwab. 2009b. Intestinal digestibility of amino acids in rumen undegraded protein: II. Distillers dried grains with solubles and fish meal. J. Dairy Sci. Submitted. doi:10.3168/jds.2008-1885

Erbersdobler, H. F., and V. Somoza. 2007. Forty years of furosine Forty years of using Maillard reaction products as indicators of the nutritional quality of foods. Mol. Nutr. Food Res. 51:423-430.

Faldet, M. A., L. D. Satter, and G. A. Broderick. 1992. Determining optimal heat treatment of soybeans by measuring available lysine chemically and biologically with rats to maximize protein utilization by ruminants. J. Nutr. 122:151-160

Finot, P. A., R. Deutsch, and E. Bujard. 1981. The extent of the Maillard reaction during the processing of milk. Prog. Food Nutr. Sci. 5:345-355
Guerra-Hernandez, E., and N. Corzo. 1996. Furosine determination in baby cereals by ion-pair reversed-phase liquid chromatography. Cereal Chem. 73:729-731.

Hurrell, R. F., and K. J. Carpenter. 1981. The estimation of available lysine in foodstuffs after Maillard reactions. Prog. Food Nutr. Sci. 5:159-176.

Kononoff, P. J., S. K. Ivan, and T. J. Klopfenstein. 2007. Estimation of the proportion of feed protein digested in the small intestine of cattle consuming wet corn gluten feed. J. Dairy Sci. 90:23772385.

Mauron, J. 1981. The Maillard reaction in food: A critical review from the nutritional standpoint. Prog. Food Nutr. Sci. 5:5-35.

Moughan, P. J., M. P. J. Gall, and S. M. Rutherfurd. 1996. Absorption of lysine and deoxyketosyllysine in an early-Maillard browned casein by the growing pig. J. Agric. Food Chem. 44:1520-1525.

Moughan, P. J., and S. M. Rutherfurd. 1996. A new method for determining digestible reactive lysine in foods. J. Agric. Food Chem. 44:2202-2209.

National Research Council. 2001. Nutrient Requirements of Dairy Cattle. 7th rev. ed. Natl. Acad. Sci. Washington D.C.

Pahm, A. A., C. Pedersen, and H. H. Stein. 2008. Application of the reactive lysine procedure to estimate lysine digestibility in distillers dried grains with solubles fed to growing pigs. J. Agric. Food Chem. 56:9441-9446.

Rutherfurd, S. M., P. J. Moughan, and L. van Osch. 1997. Digestible reactive lysine in processed feedstuffs: Application of a new bioassay. J. Agric. Food Chem. 45:1989-1994.

SAS Institute. 2001. SAS/STAT User's Guide. Version 8 ed. SAS Inst. Inc., Cary, NC.

St-Pierre, N. R. 2003. Reassessment of biases in predicted nitrogen flows to the duodenum by NRC 2001. J. Dairy Sci. 86:344-350.

Stein, H. H., M. L. Gibson, C. Pedersen, and M. G. Boersma. 2006. Amino acid and energy digestibility in ten samples of distillers dried grain with solubles fed to growing pigs. J. Anim. Sci. $84: 853-860$ 